\title{
Extracorporeal Cardiopulmonary Resuscitation in Indian Scenario: A Web-based Survey
}

\author{
Krishna M Gulla ${ }^{1}$, Tanushree Sahoo $^{2} \odot$, Suneel K Pooboni ${ }^{3} \odot$, Pranay Oza ${ }^{4}$
}

\begin{abstract}
Background: Practice and knowledge of extracorporeal cardiopulmonary resuscitation (ECPR) in an Indian setting is not known. The etiology could be multifactorial, such as lack of awareness, lack of facilities, and lack of finances. Unless we identify and rectify the underlying problems, utilization of this aspect of extracorporeal membrane oxygenation (ECMO) support would be difficult.

Materials and methods: This cross-sectional observational study was done over 6 months in three phases: (A) Formation of questionnaire/tool kit by Delphi method for 1 month (July 2019), (B) circulation of questionnaire to participants in the form of Google Forms and data collection for 2 months (August and October 2019), and (C) analysis, compilation of data, and writing the final report over 1 month (November 2019).

Results: Sixty-four participants responded. The majority of the respondents were intensivists (50\%). Only six respondents (9.5\%) had done ECPR at their center with median ECPRs per year of 2 (1-10). All ECPRs were being done in private sector hospitals. The most common indication for initiation was conventional cardiopulmonary resuscitation (CPR) for more than 10 minutes without return of spontaneous circulation (ROSC) $(n=4,66 \%)$. In all cases, the intensivists took decision for the initiation of ECPR. The rest 57 did not have the experience of ECPR at their center due to lack of equipment and experience (50\%) and financial issues (50\%).

Conclusion and clinical significance: There is a huge need to increase the awareness of the ECPR program and teams to be trained in India. We also suggest that the tertiary care medical institutions in public sector as well as the private sector that is offering critical care courses should train fellows on ECPR to employ it at times when needed to improve the outcomes of critically ill patients.

Keywords: Cardiac arrest, ECMO (Extracorporeal membrane oxygenation), Intensive care, Questionnaire.

Indian Journal of Critical Care Medicine (2021): 10.5005/jp-journals-10071-23850
\end{abstract}

\section{INTRODUCTION}

Extracorporeal cardiopulmonary resuscitation (ECPR) is deployed to support patients in refractory cardiac arrest while reversible causes are being identified or diagnosis is established, or as a bridge to transplantation. ${ }^{1}$ As per recent Extracorporeal Life Support Organization (ELSO) statistics (July 2019), out of 6,994 runs in adults where ECPR was deployed, 2,923 (41\%) survived ECPR and 2,074 (29\%) survived to discharge or transfer. Out of 4,608 pediatric ECPR runs, 2,760 (59\%) survived ECPR and 1,957 (42\%) survived to discharge or transfer. Out of 1,923 neonatal ECPR runs, 1,359 (70\%) survived ECPR and 812 (42\%) survived to discharge or transfer. ELSO recommends that it is medically futile to proceed with ECPR if cardiopulmonary resuscitation (CPR) has been unsuccessful for 30 minutes. $^{2}$ ECPR is applicable to in-hospital cardiac arrest (IHCA) or out-of-hospital cardiac arrest (OHCA) or both. Wang et al. reported the benefit of ECPR for both IHCA and OHCA patients. ${ }^{3,4}$ In India, the initiation of extracorporealmembrane oxygenation (ECMO) dates back to 2001 when it was exclusively used for postoperative cardiac cases. For the first few years, only anecdotal cases have been reported for using ECMO for reasons other than cardiac surgical cases. Since then, the use of ECMO was gradually increased over the last several years. Data from around 18 centers in India (January 2010-January 2017) where ECMO is being actively performed showed 295 cardiac ECMO cases with the survival of $57.6 \%$, 307 respiratory ECMO cases with the survival of $41.3 \%$, and 18 ECPR cases with the survival of $22.2 \%$, respectively. ${ }^{5}$ Although we progressed over the last few years in treating critically ill patients using ECMO, we still lag behind the developed nations. Currently, we depend on data and guidelines from ELSO. However, there might be a lot of regional variations in terms of indications, available
1Department of Pediatrics, All India Institute of Medical Sciences, Bhubaneswar, Odisha, India

${ }^{2}$ Department of Neonatology, All India Institute of Medical Sciences, Bhubaneswar, Odisha, India

${ }^{3}$ Department of Pediatric Critical Care Unit, Mediclinic Hospital, Abudhabi, United Arab Emirates

${ }^{4}$ Riddhivinayak Critical Care and Cardiac Centre, Malad, Mumbai, Maharashtra, India

Corresponding Author: Krishna Mohan Gulla MD; FNB; DM Associate Professor, Department of Pediatrics, All India Institute of Medical Sciences, Bhubaneswar, Odisha, Phone: +91 9582273062 e-mail: mohangulla35@gmail.com

How to cite this article: Gulla K M, Sahoo T, Pooboni S K, et al. Extracorporeal Cardiopulmonary Resuscitation in Indian Scenario: A Web-based Survey. Indian J Crit Care Med 2021;25(6):675679.

Source of support: Nil

Conflict of interest: None

technology, and economic status in India. Hence, itis the need of an hour to know about practices of ECMO in India to carry forward this life-saving technology in our scenario. As a part of this motive, we planned this study to describe the practice and knowledge about ECPR among Indian physicians.

\section{Methods}

In this cross-sectional observational study, the total duration of the study was 6 months: (A) Formation of questionnaire/tool 
kit by Delphi method for 1 month (July 2019), (B)circulation of questionnaire to participants in the form of Google Forms and data collectionfor 2 months (August and October 2019), and (C) analysis, compilation of data, and writing the final report over 1 month (November 2019).

Prior to initiation, in the first phase, a Delphi Survey was conducted in a team of five members (comprising of two ECMO specialists, two intensivists, and one perfusionist) for framing the questions for Google Form. Each member of the Delphi Survey team wasrequested to provide a set of 30 questions (multiplechoice questions with one or multiple answers) individually, and they were requested to submit the questions within the period of 2 weeks. Then, all 150 questions from five different team members were obtained, and a Web conference call meeting (by Zoom) was arranged among the team members for discussing the questions. After a combined web meeting, a consensus was reached, and the most important questions (which were common among all) and addressing the most common issues were finalized. These questions included demographic characteristics of the participants, their profession, the type of setup, their skill, and experience related to ECPR practice, etc., and some financial issues and hurdles for ECPR. The questions were pilot run in a small sample size of 5 for the next 7 days to find out any grammatical mistakes as well as mistakes in the content, which were corrected subsequently (Annexure 1).

In the second phase, we circulated the questions in the form of Google Forms to the individual emails of persons who practice intensive care (adult and pediatrics), cardiac critical are, and ECMO [e.g.,intensive care unit (ICU) fellows, intensivists, CTVS surgeons, and perfusionists]. Also, the questionnaires were shared in the WhatsApp or Telegram group of the members. In the entire duration of data collection (8 weeks), a repeat reminder was sent to participants who have not filled the form yet in every 2 weeks. Once an entry was completed and a form was submitted, the results remained locked in the Web to avoid tampering. Duplicate entry was prohibited, meaning any individual who had filled the form once wasnot allowed to fill it twice.

In the third phase, we downloaded all the responses from the participants (filled Google Forms) in the form of a combined excel sheet, and data werechecked and cleared for any possible errors thatwere clarified prior to final analysis. The identity of individual participants was never disclosed in any part of the study and as such, they were never contacted personally during the study for clarifying any query regarding filling the questionnaire.

\section{Sample Size}

As it was a web-based survey where we needed voluntary participation of participants who were supposed to spend their precious time without any reward on incentives, prior to the conduct of the study, we were expecting a dropout. Hence, keeping this information in mind as well as considering the time limitation of the study period, no prior sample size was calculated. A priori, we had decided to include all the participants who could complete the form during the data collection period.

\section{Statistical Analysis}

The data collected in the form of Google Forms were transferred to common excel sheet and analyzed using descriptive statistics [mean (SD), median (IQR), and number (\%)] subsequently. Results were provided in the form of tables wherever applicable.

\section{Ethical Clearance}

Being a web-based questionnaire, no ethical clearance was obtained prior to the study. However, consent from individual participants was obtained. Individual identities of the participants were never disclosed in any part of the study.

\section{Results}

In this web-based survey, we enrolled 64 participants. After excluding a duplicate entry, a total of 63 forms were considered for final analysis. The majority of the respondents were intensivists (adult and pediatric) constituting nearly 50\%, while only three were surgeons. Out of the entire cohort, only six participants (9.5\%) practice ECPR at their center (Table 1). All those who practice ECPR at their institute belonged to the private sector. The median caseload per year was 2 (1-10)/annum. The most common indication forinitiation was conventional CPR for more than 10 minutes without ROSC ( $n=4,66 \%)$. The common exclusion criteria cited by these participants were age $>80$ years, terminal stage malignancy, prior existing multiple organ dysfunction syndrome, severe acute neuromorbidities, and refractory infections. In all cases, intensivists took decision for the initiation of ECPR. Five people said they obtained consent prior to the procedure, and it is the intensivist who is involved in obtaining the consent. The average time for the ECMO team to be contacted was $<15$ minutes in all cases, and the time taken by ECMO team to reach ECPR deployment site was less than 30 minutes. Most (five out of six) used the peripheral femoral route for ECMO cannulation, and in all cases, conventional CPR during ECPR deployment was provided manually. In all cases, cannulation was not prepared apriori, and the procedure was conducted either in ICU $(n=3)$ or at the site of cardiac arrest $(n=3)$. Nearly $30 \%$ of the patients who underwent ECPR survived as per the survey. Most of the respondents feel the high cost of ECPR precludes its wider use with the average cost being 3,00,000 Indian Rupees (INR) (Table 2). The rest 57 respondents did not have experience of ECPR at their center: The most common reason cited was lack of equipment, experience, and financial issues (each accounting for nearly $50 \%)$. Nearly half of them $(n=27)$ thought initiation ECPR in the future wouldbe useful. Out of 57 respondents without previous experience of ECPR (Table 3), 20 respondents provided their opinion regarding the practice of ECPR, which has been summarized in Table 4. Their opinion on inclusion and exclusion criteria for ECPR was in consistent with those respondents who practice ECPR.

\section{Discussion}

In our Web-based survey, the overall practice of ECPR was low $(<10 \%)$ in the Indian scenario.ECPR is being used in adult population, that too in private sector hospitals following IHCA. Attempt for initiation of ECPR is made nearly within 15 minutes of cardiac arrest,

Table 1: Overall characteristics of respondents

\begin{tabular}{lc}
\hline Profession & Number (\%), $n=63$ \\
\hline Fellow & $17(27.0)$ \\
ECMO specialist & $7(11.1)$ \\
Perfusionist & $5(7.9)$ \\
Surgeon & $3(4.8)$ \\
Intensivist & $31(49.2)$ \\
Performing ECPR at Center & $6(9.5)$ \\
Planning to start ECPR in the near future & $30 / 57(52.6)$ \\
\hline
\end{tabular}


Table 2: Response from participants who practice ECPR

\begin{tabular}{lll}
\hline SI. No & Questions & $\begin{array}{l}\text { Number (\%) } \\
(N=6)\end{array}$ \\
\hline $1 . \quad$ & Your current position & \\
& Fellow & 0 \\
& ECMO specialist & $2(33.3)$ \\
& Perfusionist & $1(16.6)$ \\
& Surgeon & $1(16.6)$ \\
& Intensivist & $2(33.3)$ \\
2. & Place of work & \\
& Public/government sector & 0 \\
& Private sector & $6(100)$ \\
3. & If yes, you can choose multiple options & \\
& IHCA & $6(100)$ \\
& OHCA & 0 \\
& Adult age-group & $6(100)$ \\
& Pediatric age-group & $2(33.3)$
\end{tabular}

4. Median (range) number of cases performed 2 (1-10)

5. Please select your inclusion criteria

Possibly in cardiac origin $3(50)$

Witnessed cardiac arrest 2 (33.3)

CPR for more than 10 min without ROSC $\quad 4$ (66.6)

6. Please select your exclusion criteria (you

can choose multiple options) $\quad 5(83)$

Age $>80$ years $\quad 5(83)$

Terminal stage malignancy $5(83)$

Preexisting multiorgan dysfunction $\quad 5(83)$

Ventilator-dependent $>3$ months $5(83)$

Bedridden $>3$ months, not self-independent 5 (83)

infarct or severe head injury 5 (83)

Traumatic origin, uncontrollable bleeding 5 (83)

Arrest without active CPR 5 (83)

Nonwitnessed cardiac arrest

Uncontrolled infection 5 (83)

ROSC for 20 min after resuscitation without 5 (83)

repeated collapse

Patient with "DNR" orders

7. Who takes the decision to initiate ECPR?

(You can choose multiple options)

Admitting physicians 2 (33.3)

Intensivist $6(100)$

Surgeon $3(50)$

Family $3(50)$

All 1 (16.7)

8. Do you take consent for ECPR?

Yes $5(83)$

No 1 (16.7)

9. Who takes the consent?

Admitting physicians $\quad 0(0)$

Intensivist $\quad 5$ (83)

Surgeons $0(0)$

Nursing staff 2 (33.3)

ECMO specialists 3 (50)

10. After how much time of CPR, ECMO team is

usually contacted?

$<5$ min

$0(0)$

$10 \mathrm{~min}$

$4(67)$

15 min

$2(33.3)$
Table 2: (Continued)

\begin{tabular}{lll}
\hline Sl. No & Questions & $\begin{array}{l}\text { Number }(\%) \\
(N=6)\end{array}$ \\
\hline $20 \mathrm{~min}$ & $0(0)$ \\
$30 \mathrm{~min}$ & $0(0)$ \\
$>30 \mathrm{~min}$ & $0(0)$
\end{tabular}

11. How much time taken for ECMO team

to arrive to initiate ECPR in your center?

$<10 \mathrm{~min}$

$10-20 \mathrm{~min}$

20-30 min

$3(50)$

$>30 \mathrm{~min}$

12. Site of cannulation you use for ECPR

(you can choose multiple options)

Central

Peripheral femoral

Peripheral neck

13. What is the average no.of flow time (time from the cardiac arrest to initiation of manual (PR) in your setup?

$<5$ min $5(83)$

5-10 min $\quad 1(16.7)$

10-15 min 0

$>15$ min 0

14. What is the average ischemia time (cardiac arrest to ECMO initiation)?

$<30$ min $4(66.7)$

30-60 min $2(33.3)$

60-90 min 0

$>90$ min 0

15. How does the conventional CPR take

place during cannulation?

Manual

Mechanical

$6(100)$

Both

0

16. Do you use therapeutic hypothermia

after establishing ECPR?

Yes $4(66.7)$

No $1(16.7)$

May be 1 (16.7)

17. Do you have any specific time for ECPR?

$1(16.7)$

Nighttime

None holiday

0

18. Location of ECPR in IHCA(you can

choose multiple options)

ICU $5(83)$

OT 4 (66.7)

Cath Lab $\quad 4$ (66.7)

Wards

Imaging room (CT/MRI)

0

0

19. Where do you do cannulation for IHCA?

Site of cardiac arrest

$\mathrm{ICU}$

Operation theater 0

Wards

0

20. Who does cannulation in IHCA?

Surgeon

Intensivist

ECMO specialists 
Table 2: (Continued)

\begin{tabular}{lll}
\hline SI. No & Questions & $\begin{array}{l}\text { Number (\%) } \\
(N=6)\end{array}$ \\
\hline 21. & $\begin{array}{l}\text { Do you keep primed circuits ready in antici- } \\
\text { pation of ECPR round the clock? }\end{array}$ & 0 \\
& Yes & $6(100)$ \\
& No & 0 \\
& May be & \\
22. $\quad \begin{array}{l}\text { What is the average percentage of patients } \\
\text { who survived to discharge at your center? }\end{array}$ & $30 \%$ \\
23. $\quad \begin{array}{l}\text { What is the percentage of intact neurologi- } \\
\text { cal survival of ECPR patients at your center? }\end{array}$ & - \\
24. $\quad \begin{array}{l}\text { What is the average cost of ECPR at your } \\
\text { center? }\end{array}$ & Rs. 3,00,000 \\
\end{tabular}

Table 3: Response from participants without experience of ECPR

\begin{tabular}{lll}
\hline SI. No. & Questions & Number 57 (\%) \\
\hline 1. & Your current position & $N=57$ \\
& a. Fellow & $17(29.8)$ \\
& b. ECMO specialist & $5(8.7)$ \\
& c. Perfusionist & $4(7)$ \\
d. Surgeon & $2(3.5)$ \\
& e. Intensivist & $29(50.8)$ \\
2. & Place of work & $N=57$ \\
& a. Public/government sector & $12(21.0)$ \\
& b. Private sector & $45(79.0)$ \\
3. & Why don't you perform ECPR? & \\
& a. Lack of experience/knowledge & $28(49.1)$ \\
& b. Lack of team round the clock & $13(22.8)$ \\
& c. Lack of belief in ECPR & $2(3.5)$ \\
& d. Lack of equipment & $8(14.0)$ \\
& e. Financial issues & $8(14)$ \\
& f. Lack of management commitment & $14(24.5)$ \\
Are you planning to initiate the & \\
EPCR program in the unit? & $30(52.6)$ \\
& a. Yes & $27(47.4)$ \\
\hline
\end{tabular}

and manual CPR is performed while cannulation for ECPR. The most common route of cannulation is theperipheral femoral route.

In India, ECPR is still in an infancy stage. Till now, there is no available literature on the use and practice of ECPR in India. As compared to the western scenario, the practice of ECPR is very suboptimal. There are a number of barrierstoits poor uptake: financial issues and lack of adequate training being the most common ones. The knowledge, awareness, and belief in ECPR arealso probablylow among intensivists, surgeons, and perfusionists in India. With the vast expansion inthe field of critical care in India and improved overall financial well-being of the people, and upcoming financial schemes (Insurance based, Govt programs like Ayushmann Bharat), we expect an increase in uptake of this life-saving technology in the future. There is an urgent need for creating awareness among intensivists as well as the general public for the benefits of ECPR by incorporating the topic as part of a training forfellows enrolled in critical care program in India.
Table 4: Response of persons who are not performing ECPR but wanted to express their view on ECPR

\begin{tabular}{ll}
\hline 1. If yes, you can choose multiple options & $\mathrm{N}=12$ \\
a. IHCA & $10(83)$ \\
b. OHCA & $3(25)$ \\
c. Adult age-group & $7(58)$ \\
d. Pediatric age-group & $9(75)$ \\
2. $\quad$ Please select your inclusion criteria & $\mathrm{N}=19$ \\
a. Possibly in cardiac origin & $10(53)$ \\
b. Witnessed cardiac arrest & $14(74)$ \\
c. CPR for more than 10 min without ROSC & $10(53)$ \\
Please select your exclusion criteria (you can & \\
choose multiple options) & $\mathrm{N}=19$ \\
a. Age >80 years & $17(90)$ \\
b. Terminal stage malignancy & $18(95)$ \\
c. Preexisting multiorgan dysfunction & $14(74)$ \\
d. Ventilator-dependent >3 months & $16(84)$ \\
e. Bedridden >3 months, not self-independ- & $16(84)$ \\
ent & $17(90)$ \\
f. Acute/active intracranial hemorrhage or & \\
infarct or severe head injury & 0 \\
g. Traumatic origin, uncontrollable bleeding & 0 \\
h. Arrest without active CPR & 0 \\
i. Nonwitnessed cardiac arrest & $11(58)$ \\
j. Uncontrolled infection & 0 \\
k. ROSC for 20 min after resuscitation without & 0 \\
repeated collapse & 0 \\
I. Patient with “DNR" order & \\
m. Others &
\end{tabular}

4. Who should take the decision to initiate

ECPR? (You can choose multiple options) $\quad \mathrm{N}=15$

a. Admitting physicians 2

b. Intensivist $\quad 5$

c. Surgeon 0

d. Family 2

e. All 8

5. Should you take consent for ECPR? $\quad N=15$

a. Yes 11

b. No 2

c. May be 2

6. Who should take the consent? $\quad N=15$

a. Admitting physicians 6

b. Intensivist $\quad 11$

c. Surgeons 3

d. Nursing staff 3

e.ECMO specialists 5

7. After how much time of CPR, should ECMO

team be contacted? $\quad N=13$

a. $<5 \mathrm{~min} \quad 4$

b. $10 \mathrm{~min} \quad 5$

c. $15 \mathrm{~min} 2$

d. $20 \mathrm{~min}$

e. $30 \mathrm{~min}$

f. $>30 \mathrm{~min} \quad 1$

8. How much time it should take for ECMO team to arrive to initiate ECPR in your center? $\quad N=10$

a. $<10 \mathrm{~min} 3$

b. $10-20 \mathrm{~min} \quad 1$

c. $20-30 \mathrm{~min} \quad 3$

d. $>30 \mathrm{~min} \quad 3$

(Continued) 


\section{Table 4: (Continued)}

9. Which site should be preferred for cannulation for ECPR? (You can choose multiple options)

a. Central

b. Peripheral femoral

c. Peripheral neck

10. What should be the average no. of flow time (time from the cardiac arrest to initiation of manual (PR) in your setup?

a. $<5 \mathrm{~min}$

b. $5-10 \mathrm{~min}$

c. $10-15 \mathrm{~min}$

d. $>15 \mathrm{~min}$

11. What should be the average ischemia time (cardiac arrest to ECMO initiation)?

a. $<30 \mathrm{~min}$

b. $30-60 \mathrm{~min}$

c. $60-90 \mathrm{~min}$

d. $>90 \mathrm{~min}$

12. How should the conventional CPR take place during cannulation?

a. Manual

b. Mechanical

c. Both

13. Should you use therapeutic hypothermia after establishing ECPR?

a. Yes

b. No

c. May be

14. When should ECPR be performed?

a. Daytime

b. Nighttime

c. Any time

d. None holiday

15. What should be the ideal location of ECPR in IHCA?

a. ICU

b. OT

c. Cath lab

d. Wards

e. Imaging room (CT/MRI)

16. Where should you do cannulation in IHCA? $\quad \mathrm{N}=11$

a. Site of cardiac arrest $3(27)$

b. ICU

c. Operation theater

d. Wards

17. Who should do cannulation in IHCA?

a. Surgeon

b. Intensivist

c. ECMO specialists

18. Should primed circuits be ready in anticipation of ECPR round the clock?

a.Yes

b.No

c.May be
As an increasing number of cardiac surgeries (adult and pediatric) are being performed in India, we would like to encourage cardiac ICUsto incorporate ECPR as part of their packages in cardiac ICUs so that for slightly increased cost, both the patients and the hospital can get benefited by improving the patient's outcomes.

\section{Conclusion}

There is a huge need to increase the awareness of the ECPR program and teams to be trained in India. We also suggest that the tertiary care medical institutions in the public sector as well as the private sector that isoffering critical care courses should train fellows, cardiac surgeons, and perfusionists on ECPR to employ it at times when needed to improve the outcomes of critically illpatients. Policies, financial packages as well as counseling teams should be made available to help with safeguarding the interests of patients as well as their families.

\section{ACKnOWLedgments}

We are thankful to all participants who filled the questionnaire and provided their valuable inputs.

\section{OrCID}

Krishna M Gulla 구 http://orcid.org/0000-0001-5031-5754

Tanushree Sahoo (1) https://orcid.org/0000-0003-0815-8710

Suneel K Pooboni (1) https://orcid.org/0000-0001-6855-3611

\section{References}

1. de Caen AR, Berg MD, Chameides L, Gooden CK, Hickey RW, Scott HF, et al. Part 12: pediatric advanced life support: 2015 American Heart Association guidelines update for cardiopulmonary resuscitation and emergency cardiovascular care. Circulation 2015;132 (18 Suppl. 2): S526-S542. DOI: 10.1161/CIR.0000000000000266.

2. Extracorporeal Life Support Organization (ELSO) patient specific supplements to the ELSO general guidelines; 2009. Available at: http://square.umin. ac.jp/jrcm/pdf/ecmo/ecmotext12.pdf [Accessed July 23, 2016].

3. Wang CH, Chou NK, Becker LB, Lin JW, Yu HY, Chi NH, et al. Improved outcome of extracorporeal cardiopulmonary resuscitation for outof-hospital cardiac arrest - a comparison with that for extracorporeal rescue for in-hospital cardiac arrest. Resuscitation2014;85(9):1219-1224. DOI: 10.1016/j.resuscitation.2014.06.022.

4. Tan BK. Extracorporeal membrane oxygenation in cardiac arrest. Singapore Med J 2017;58(7):446-448. DOI: 10.11622/smedj.2017058.

5. Pooboni S, Goyal V, Oza P, Kapoor PM. ECMO challenges and its future: Indian scenario. J Card Crit Care TSS 2017;1(2):89-94. DOI: $10.1055 / \mathrm{s}-0038-1626672$. 